\title{
Evolution of the StreamHash hash function family
}

\author{
Michał Trojnara* \\ Faculty of Electronics and Information Technology, Warsaw University of Technology, \\ ul. Nowowiejska 15/19, 00-665 Warszawa, Poland
}

\begin{abstract}
This paper describes the evolution of StreamHash cryptographic hash function family proposed by the author. The first member of the StreamHash family was StreamHash (now called StreamHash1) function, accepted for the first round of SHA-3 competition organized by the US government standards agency $\mathrm{NIST}^{\dagger}$. The competition has been started in order to select a new SHA-3 standard as the successor of SHA-2 family of cryptographic hash functions. Function StreamHash2 mostly addresses security weaknesses identified during the SHA-3 competition, while the sketch of function StreamHash3 attempts to improve resistance to side-channel attacks and performance properties. The paper starts with an overview of basic properties of cryptographic hash functions followed by the description of the StreamHash family design principles and its basic structure. Subsequent sections illustrate the way each subsequent function uses lessons learnt while designing and testing the previous one.
\end{abstract}

\section{Overview of the StreamHash family}

\subsection{Cryptographic hash functions}

The cryptographic hash function is a deterministic function that transforms arbitrary blocks of data into fixed-size values. The hash value for any given

*E-mail address: Michal.Trojnara@mirt.net

${ }^{\dagger}$ National Institute of Standards and Technology 
message can be efficiently computed, i.e. $h(m)$ value can be easily computed for any given message $m$.

The following main security properties are required:

(1) It is not practically feasible to find a message transformed into a given hash (also known as preimage), i.e. for any given $h(m)$ value it is infeasible to find a corresponding message $m$. This property is called preimage resistance.

(2) It is not practically feasible to modify a message without changing its hash, i.e. for any given $m_{1}$ message it is infeasible to find another $m_{2}$ message (also known as the second preimage) such that $h\left(m_{1}\right)=$ $h\left(m_{2}\right)$. This property is called second preimage resistance.

(3) It is not practically feasible to find two different messages with the same hash, i.e. it is infeasible to find two different messages $m_{1}$ and $m_{2}$ (also known as collision) such that $h\left(m_{1}\right)=h\left(m_{2}\right)$. This property is called collision resistance.

Some auxiliary properties are also often required:

(1) The hash function output should be indistinguishable from random numbers, so they can be used as a foundation for keystream generators. For example SSL and TLS [1] protocols use a mix of MD5 and SHA-1 to produce a sufficient number of master secret bits from an initial premaster secret and exchanged random values.

(2) The function should be resilient to length-extension attacks: given $h\left(m_{1}\right)$ and $\operatorname{len}\left(m_{1}\right)$, but not $m_{1}$ itself, it should not be practically feasible to calculate $h\left(m_{1} \|\right.$ padding $\left.\| m_{2}\right)$. This property can be used to break naive authentication schemes based on the hash functions. The $\mathrm{HMAC}^{\ddagger}[\mathbf{2}]$ construction works around these problems.

Practical infeasibility should not be confused with theoretical computational complexity measures such as time or memory consumption. Theoretical measures cover either best, worst or average complexity. For cryptographic applications it is acceptable to violate any of the above properties as long as the probability of failure is negligible.

Cryptographic hash functions are often mistaken for checksums such as CRC32, only designed to detect accidental and not intentional modification of data.

Applications of cryptographic hash functions include:

- Digital signatures.

- Message authentication codes (MACs).

- User or device authentication.

${ }^{\ddagger}$ keyed-Hash Message Authentication Code 


\subsection{Design rationale}

Commonly used cryptographic hash functions are based on the Merkle-Damgård construction. The input message is processed in blocks. The message needs to be padded, so the length of the padded message is a multiple of the block size. Further processing is performed with a compression function. The function takes two inputs: a chaining variable and a message block. Compression function outputs the next value of the chaining variable. Each block of a padded message is iteratively processed with a compression function, starting with a predefined initial value of the chaining variable.

Compression function is performed in several rounds in order to provide required cryptographic properties. Each round only performs non-trivial (e.g. non-linear) operations on a subset of the chaining variable, while the remaining part is merely shifted. This is why multiple rounds are needed to achieve the avalanche effect, so that every bit of output depends on every bit of input of the compression function.

The approach of the StreamHash family is completely different. Instead of achieving the avalanche effect with multiple rounds, it directly updates the state vector on each octet of the input stream.

The structure of the StreamHash family is based on a well-known problem of solving a set of non-linear equations or $\mathrm{CSP}^{\S}$. Common algorithms for solving CSPs [3] include backtracking, constraint propagation, and local search. The StreamHash family is designed, so that these algorithms cannot be applied. This property is ensured by the clear separation of the constraints. Solving a subset of all constraints does not make solving remaining constraints any easier.

No security proof is provided for the StreamHash family. Specifically no reduction from CSP or any other NP-complete problem has been demonstrated.

\subsection{NLF transformation}

The main building block of StreamHash family is a fast non-linear transformation NLF (Non-Linear Function).

Figure 1 illustrates inputs and outputs of the $N L F$ transformation.

$i$ - state vector index

state $_{i}$ - previous state vector element

state $_{i+1}-$ next state vector element

$c$ - input octet index (added in StreamHash2)

$b_{c}-$ input octet (StreamHash1, StreamHash2) or word (StreamHash 3)

$r_{c}-P R N G$ value (added in Streamhash2)

\footnotetext{
${ }^{\S}$ Constraint Satisfaction Problem
} 


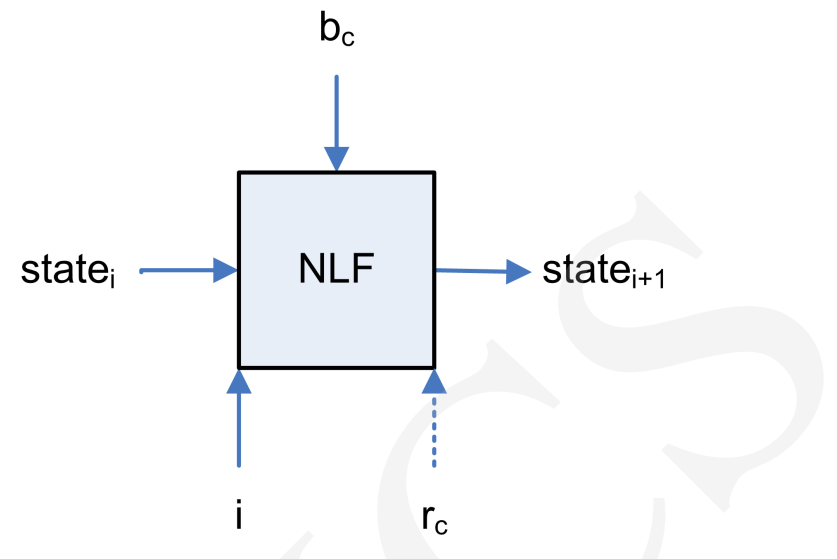

Fig. 1. NLF function.

\subsection{Structure}

See Figure 2 for the diagram of the StreamHash family structure.

A separate transformation is also applied in the finalization phase. Finalization is designed to prevent the length-extension attacks and to improve statistical properties of the output.

\subsection{Advantages of the StreamHash family}

The main advantages of the StreamHash family are:

- Clear and easy to analyze design.

- Negligible performance impact of machine endianness.

- High performance on 8-bit and 16-bit architectures.

- Easy to parallelize internal structure with theoretical performance up to a single clock cycle per input octet.

- Fast finalization resulting in low latency. This property is extremely important in real-time (e.g. multimedia) applications.

- Fast finalization resulting in high throughput for short messages.

- Minimal size of code, important for embedded systems.

- Minimal size of variables, important for embedded systems.

- Low size of static data.

- Scalability to use any multiple of 32 bits as the hash value length.

\subsection{Limitations of the StreamHash family}

The mathematical background is also not well studied in cryptographic applications. While this is not a direct weakness, extensive cryptanalysis is essential to trust a cryptographic primitive. 


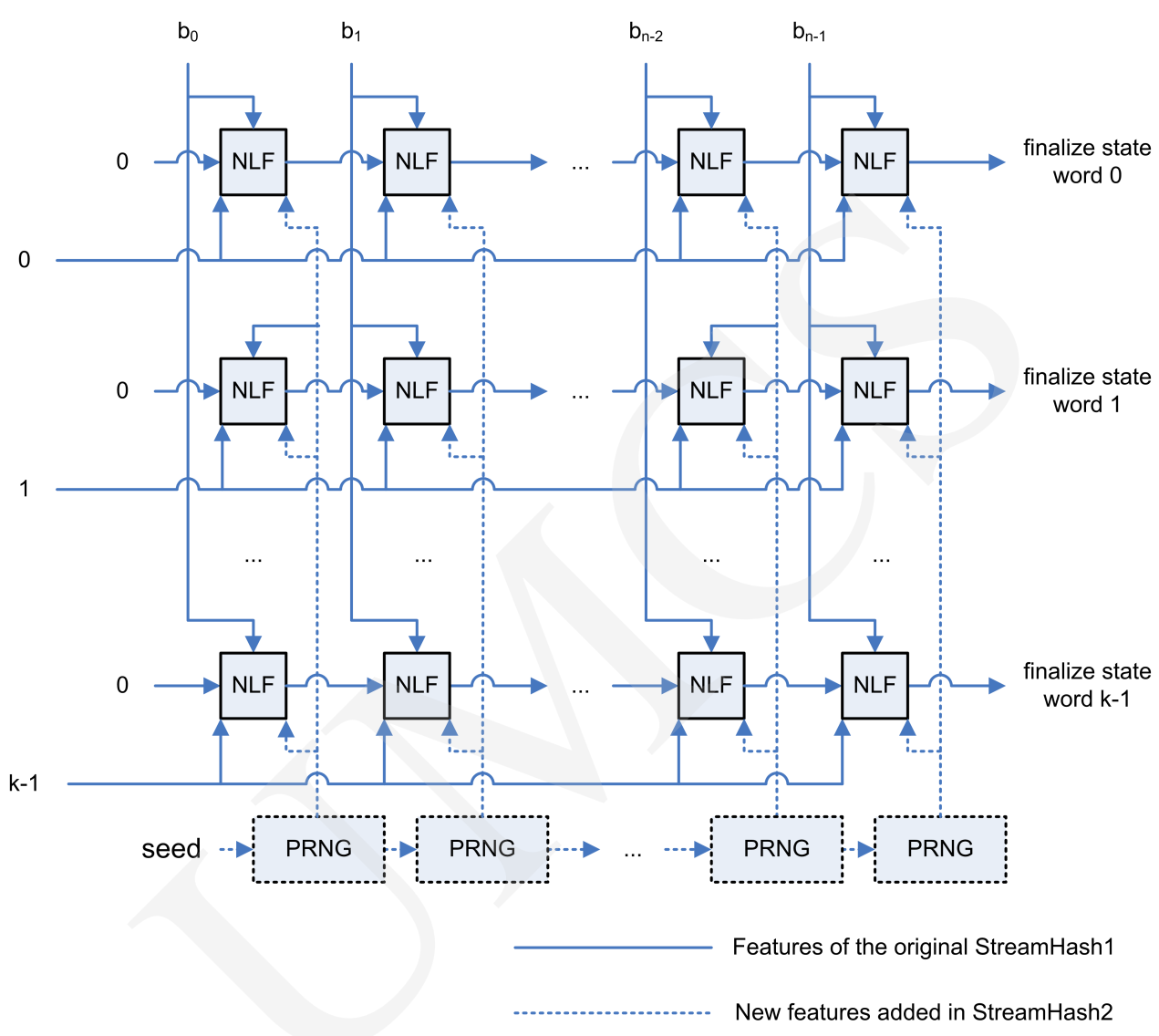

Fig. 2. StreamHash structure.

\section{StreamHash1 function}

\subsection{Motivation}

The StreamHash[4] (now called StreamHash1) algorithm was accepted for the first round of SHA-3 competition organized[5] NIST.

The main motivation for StreamHash1 was to demonstrate security of performance properties of the StreamHash family. The function was designed to be as simple as possible in order to simplify its cryptoanalysis. Specifically, no constants or transformations were included without a clear security rationale.

As an early and immature design, StreamHash suffered from severe security weaknesses. 


\subsection{State data}

StreamHash1 state structure consists of:

- A vector of 32-bit values to hold the state for all processed octets, hereafter referred to as the state vector;

- The value of remaining bits in the last input data octet if it is not full; and

- The number $\{0,1, \ldots 7\}$ of remaining bits in the last input data octet.

The length of the state vector is equal to the message digest size divided by 32 , i.e. 7 for 224-bit digest, 8 for 256-bit digest, 12 for 384-bit digest, and 16 for 512-bit digest.

At initialization the state vector is set to zero.

\subsection{State update algorithm}

StramHash2 NLF transformation works by adding (modulo $2^{32}$ ) an $S$-BOX output to the state vector value. The $S$ - $B O X$ index is computed as:

$$
L S B\left(\text { state }_{i}\right) \oplus b \oplus i
$$

The resulting formula to update a state vector value for the index $i$ is:

$$
\text { state }_{i} \leftarrow \text { state }_{i} \oplus S-B O X\left[L S B\left(\text { state }_{i}\right) \oplus b \oplus i\right]
$$

Any remaining input data bits (for input size not being a multiple of 8 bits), and the number of these bits are both saved within the state structure.

Figure 3 illustrates the internal structure of the StreamHash1 NLF transformation.

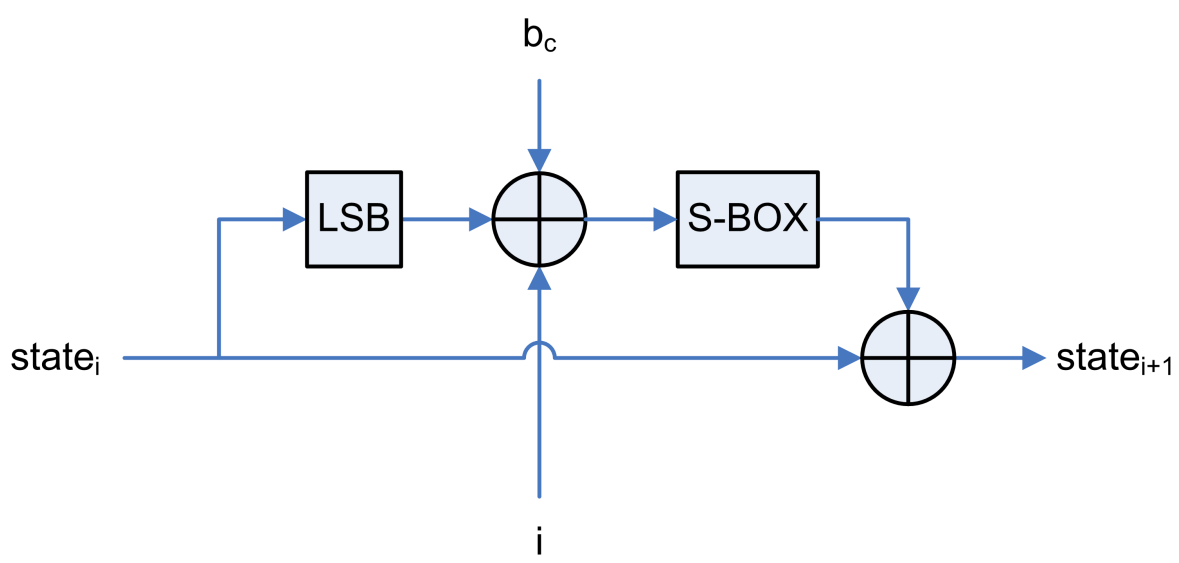

Fig. 3. NLF Function of StreamHash1. 


\subsection{Structure of $S-B O X$}

StreamHash $S$-BOX is based on AES $S$-BOX. The formula to compute the 32-bit $S$ - $B O X$ value for the index $i$ is:

$$
s(i) \vee(s(s(i)) \ll 8) \vee(s(s(s(i))) \ll 16) \vee(s(s(s(s(i)))) \ll 24)
$$

The content of the StreamHash $S-B O X$ computed using the above formula is listed in Table 1.

\subsection{Cryptanalysis}

The third-party cryptanalysis is available for the StreamHash1 function, the first function of the StreamHash family.

Dmitry Khovratovich and Ivica Nikolić from University of Luxembourg reviewed cryptographic properties of StreamHash [6]. Joux attack [7] was applied with the theoretical complexity of $\frac{n}{2} 2^{n / 4}$ for finding collisions and $\frac{n}{2} 2^{n / 2}$ for finding preimages.

Tor E. Bjorstad, a PhD student of Computer Science, University of Bergen, Norway implemented [8] a practical collision attack against the StreamHash1 function.

\section{StreamHash2 function}

\subsection{Motivation}

The StreamHash2 algorithm was designed to address identified weaknesses of the original StreamHash1 function.

\subsection{Algorithm updates}

The following changes were implemented in the StreamHash2 function compared to the original StreamHash1:

- $N L F$ transformation was modified with a 32-bit output of $P R N G^{\|}$in order to prevent from the re-use of any identified collision of a single state word.

- $\oplus$ operation was replaced with $\boxplus$ (addition modulo $2^{32}$ ) in order to propagate changes between the four octets of the 32-octet state word.

- Finalization phase was updated to improve resistance against lengthextension attacks and statistical properties.

The StreamHash2 state structure was extended with:

- 64 bits of PRNG state;

\footnotetext{
『Advanced Encryption Standard

"Pseudo-Random Number Generator
} 
Pobrane z czasopisma Annales AI- Informatica http://ai.annales.umcs.pl

Data: 26/04/2023 12:31:55

Table 1. StreamHash2 $S$ - $B O X$

\begin{abstract}
760ffb63 74ca107c 8ee6f577 54fd217b 5ca789f2 b5d27f6b 25c2a86f 3624a6c5 $89 f 20430$ ca107c01 88978567 32a1f12b 87eabbfe 62ab0ed7 acaa62ab c5073876 4f9274ca ff7d1382 78c1ddc9 4716ff7d 61d82dfa c01fcb59 e1e0a047 43648cf0 e52a95ad 005248d4 cd803aa2 4eb679af a41dde9c e23b49a4 01094072 bff4bac0 66d3a9b7 b72054fd 4486dc93 4568f726 7f6b0536 5e9d753f 6e4568f7 6db34bcc 95ad1834 a86f06a5 9635d9e5 2332a1f1 670aa371 dfef61d8 b4c6c731 1fcb5915 a789f204 8db4c6c7 68f72623 c7312ec3 2a95ad18 d0609096 d27f6b05 506cb89a 24a6c507 c1ddc912 7abdcd80 5a4698e2 721ee9eb b34bcc27 b89a37b2 585e9d75 107c0109 8bceec83 0aa3712c 803aa21a b679af1b b9db9f6e e4aebe5a f8e1e0a0 fb630052 4698e23b 2c42f6d6 eb3c6db3 6f06a529 138211 e3 cb59152f 8acf5f84 fc55ed53 37b23ed1 offb6300 b0fc55ed d3a9b720 94e7b0fc 9be8c8b1 c912395b f577026a bac01fcb 69e4aebe ddc91239 42f6d64a 06a5294c 77026a58 f37e8acf d15170d0 Ob9edfef 8191acaa 38760ffb 3aa21a43 8211e34d 312ec333 c4889785 db9f6e45 28ee99f9 e6f57702 d5b5d27f 55ed5350 1ee9eb3c 56b9db9f 3f25c2a8 b23ed151 85670aa3 7c010940 738f738f 5f844f92 6a585e9d a6c50738 198ee6f5 e34d65bc 152f4eb6 395b57da 2054fd21 9274ca10 a04716ff 0ed70df3 03d5b5d2 da7abdcd eabbfe0c 16ff7d13 3d8bceec 7e8acf5f 1cc48897 79af1b44 648cf017 de9c1cc4 d64a5ca7 d70df37e 4bcc273d a21a4364 a5294c5d 5248d419 8f738f73 $5170 d 060$ bbfe0c81 cf5f844f 1b4486dc 86dc9322 35d9e52a 70d06090 9c1cc488 aebe5a46 183428ee 53506cb8 d82dfa14 49a41dde 026a585e a1f12b0b b156b9db 41f8e1e0 f7262332 bdcd803a 9785670a 98e23b49 c2a86f06 6b053624 f6d64a5c 753f25c2 c33366d3 0c8191ac 91acaa62 fe0c8191 d9e52a95 99f969e4 2f4eb679 932294e7 149be8c8 6cb89a37 e9eb3c6d 294c5d8d 217b03d5 59152f4e 3366d3a9 ed53506c e8c8b156 3008bff4 f01787ea 11e34d65 5b57da7a f969e4ae f2043008 08bff4ba 4d65bc78 9d753f25 c6c7312e 1dde9c1c 053624a6 4c5d8db4 5d8db4c6 fa149be8 bc78c1dd $844 f 9274$ f4bac01f 3c6db34b 57da7abd cc273d8b 0df37e8a 3ed15170 9a37b23e 7b03d5b5 2ec33366 63005248 fd217b03 712c42f6 aa62ab0e 9edfef61 $6090963512395 \mathrm{~b} 57$ c8b156b9 af1b4486 65bc78c1 3b49a41d f12b0b9e $8341 \mathrm{f} 8 \mathrm{e} 1$ ec8341f8 be5a4698 7d138211 ee99f969 909635d9 48d4198e dc932294 2dfa149b 0940721e 8cf01787 40721ee9 273d8bce e7b0fc55 ad183428 2b0b9edf 1a43648c 262332a1 4a5ca789 ab0ed70d 043008bf d4198ee6 a3712c42 9f6e4568 ceec8341 3428ee99 ef61d82d 0738760f 2294e7b0 a9b72054 1787eabb e0a04716
\end{abstract}

The improved formula to update a state vector value for the index $i$ is:

$$
\text { state }_{i} \leftarrow \text { state }_{i} \boxplus S-B O X\left[L S B\left(\text { state }_{i}\right) \oplus b \oplus i\right] \boxplus r_{c}
$$

StreamHash2 shares all other parts of the StreamHash2 design described above, e.g. the $S$ - $B O X$ table.

Figure 4 illustrates the internal structure of the StreamHash2 NLF transformation. 


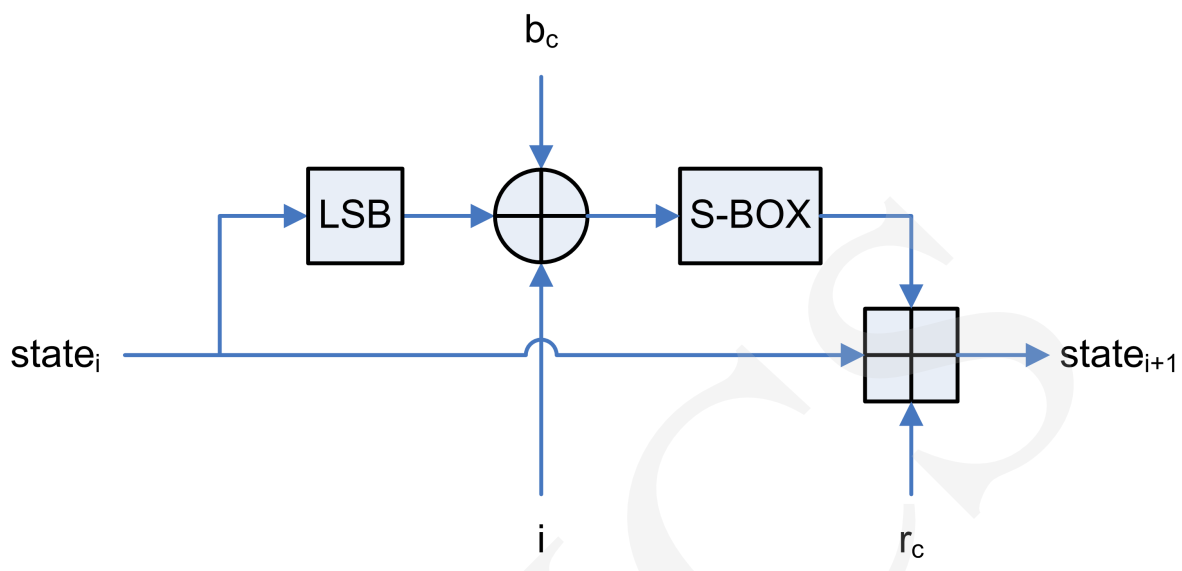

Fig. 4. NLF Function of StreamHash2.

\subsection{Pseudo-random number generator}

The StreamHash2 function uses a 64-bit version of the pseudo-random number generator Xorshift [9] as its $P R N G$ transformation. The generator provides the period of $2^{64}-1$. $P R N G$ is not expected to be cryptographically secure, and security of StreamHash2 is not based on the $P R N G$ properties other than its period.

The following algorithm is used to generate each 32-bit value of $r_{c}$ :

(1) $s \leftarrow s \oplus(s \ll 13)$.

(2) $s \leftarrow s \oplus(s \gg 7)$.

(3) $s \leftarrow s \oplus(s \ll 17)$.

(4) Return $r_{c}$ as the least significant 32 bits of $s$.

The 64-bit $P R N G$ state $s$ is initialized with the seed value of 88172645463325252 . This starting value is a constant recommended by the author of the Xorshift algorithm.

\subsection{Identified limitations of StreamHash2}

Identified disadvantages of StreamHash2 are mostly the result of $S$-BOX lookup:

- Side-channel attacks[10] on multiasking software implementations based on the CPU cache timings.

- Not possible to compute with the SIMD** instructions on x86 architecture.

- Expensive hardware implementation (high number of gates).

${ }^{* *}$ Single Instruction, Multiple Data 
- $1 \mathrm{~KB}$ of static data, although it can be reduced to 256 octets with a reasonable performance trade-off.

\section{Plans for StreamHash3 function}

\subsection{Motivation}

Daniel J. Bernstein demonstrated[10] a practical side-channel attack on the AES algorithm. The attack leverages a weakness of the AES non-linear transformation based on $S-B O X$. Multiple processes running on the same physical machine several resources of the CPU including memory caches. It is possible to force another process to perform cache hit or cache miss depending on the $S$ - $B O X$ lookup offset. With accurate time measurements it is possible to infer secret data and subsequently to compute encryption key. The same weaknesses could be used to find preimages of the StreamHash2 algorithm.

Initially, $S$ - $B O X$ appeared to be a perfect source of non-linearity for the StreamHash family. It seemed to be extremely fast, as $S$ - $B O X$ lookup is implemented with a single CPU instruction. Code profiling tests performed by the author of this paper revealed that a significant amount of CPU time is spent on the lookup instruction, as its lookups cannot be solely computed on registers.

It is also not practical to use the $S$ - $B O X$ indices longer than 8 bits for implementations with limited hardware resources. 8-bit $S$-BOX indices, in turn, only allow StreamHash2 to process one octet of input data at a time.

The use of $S-B O X$ es is not practical on low-end implementations. For low-end 8-bit CPUs $1 \mathrm{~KB}$ of static data may represent a substantial amount of memory. The $S$ - $B O X$ included in the previous StreamHash family members can, however, be computed on the fly, reducing memory usage with a reasonable performance trade-off.

This issue gets much worse for low-end hardware implementations. For lowpower hardware (e.g. RFID ${ }^{\dagger \dagger}$ tokens) the number of gates required to implement the $S$-BOX of StreamHash2 could be unacceptable.

\subsection{Proposed solution}

The solution for the planned StreamHash3 is to replace $S$-BOX es with the constructions based on shifts $(\ll$ and $\gg)$ and modular addition $(\boxplus)$ should allow to process input stream word-by-word instead of octet-by-octet, and to implement non-linearity with the SIMD instructions.

As a result, it may be possible to achieve StreamHash3 performance as good as the performance of StreamHash2, or even better.

\footnotetext{
${ }^{\dagger \dagger}$ Radio-frequency identification
} 


\subsection{Support of the $\mathrm{x} 86 \mathrm{CPU}$ architecture}

The following instructions, operating on the sets of 32-bit words, could be used on x86 architecture:

PSLLD - Packed Shift Left Logical $(\ll)$

PSRLD - Packed Shift Right Logical ( $\gg)$

PADDD - Packed Add $(\boxplus)$

The number of simultaneously processed words depends on the SIMD word size available on the specific architecture [11]. The following SIMD register is available on the x86-compatible CPUs:

MMX - 864 -bit registers $m m_{0}-m m_{7}$.

SSE2 - 8128-bit registers $x m m_{0}-x m m_{7}$ in 32-bit mode, and 16128 -bit registers $x m m_{0}-x m m_{15}$ in 64-bit mode.

AVX - 256-bit registers ymm $y_{0}-y m m_{15}$ available. The first CPUs supporting AVX architecture are Intel Sandy Bridge (first released on 9 January 2011) and AMD Bulldozer (scheduled for release on Q2 2011).

The SIMD instructions would allow to simultaneously process 2 (for MMX), 4 (for SSE2) or 8 (for AVX) 32-bit StreamHash3 state words.

\section{Conclusions}

Practical attacks against MD5 [12] and SHA-1 [13] suggest that collision resistance is the most serious threat to cryptographic hash functions. The StreamHash family was designed specifically to deal with this threat.

The whole StreamHash family can be effortlessly scaled to use any multiple of 32 bits as the state vector size. Applications of this property include not just upscaling for improved security, but also downscaling for the applications with reduced security requirements, e.g. lightweight cryptography. These applications can benefit from fast finalization of the StreamHash family, as well as the reduced number of gates achieved by removing $S-B O X$ while designing the StreamHash3 function.

Growing popularity of lightweight cryptography is driven by the increasing number of RFID tags as well as battery-powered wireless sensor agents. Currently many of these devices use plaintext communication protocols in order to reduce circuit chip size/cost and power consumption. StreamHash3 could be used as an efficient cryptographic hash function to implement HMAC-based security layer for these protocols. 


\section{Symbols}

$\boxplus \quad$ arithmetic unsigned addition modulo $2^{32}$

$\oplus \quad-$ bitwise exclusive disjunction, also called XOR (EXclusive Or)

$\checkmark$ - bitwise $O R$ operator

$\ll$ - bitwise SHIFT LEFT operator

$\gg$ - bitwise SHIFT RIGHT operator

$\leftarrow-$ substitution

$\|$ - concatenation of octet strings

$S$ - $B O X[x]$ - table lookup returns the value at the position $x$ of table $S$ - $B O X$

$L S B(x)$ - least significant octet of $x$

\section{References}

[1] Dierks T., Allen C., RFC 2246: The TLS protocol version 1, January (1999), ftp://ftp.internic.net/rfc/rfc2246.txt

[2] Krawczyk H., Bellare M., Canetti R., RFC 2104: HMAC: Keyed-hashing for message authentication, February (1997), ftp://fp.internic.net/rfc/rfc2104.txt

[3] Garey M. R., Johnson D. S., Computers and Intractability; A Guide to the Theory of NP-Completeness, W. H. Freeman \& Co., New York, NY, USA (1990).

[4] Trojnara M., Streamhash algorithm specifications and supporting documentation, http://csrc.nist.gov/groups/ST/hash/sha-3/Round1/documents/StreamHash.zip (2008).

[5] NIST. First round candidates, http://csrc.nist.gov/groups/ST/hash/sha-3/Round1/submissions_rnd1.html

[6] Khovratovich D., Nikolić I., Cryptoanalysis of streamhash, http://lj.streamclub.ru/papers/hash/streamhash.pdf (2009).

[7] Joux A., Multicollisions in iterated hash functions, application to cascaded constructions, In Matthew K. FFranklin, editor CRYPTO 3152 of LNCS (2004): 306.

[8] Bjorstad T. E., Collision for streamhash, http://ehash.iaik.tugraz.at/uploads/7/7b/Streamhash.txt (2009).

[9] Marsaglia G., Xorshift rngs, Journal of Statistical Software 8(14) (2003): 1; http://www.jstatsoft.org/v08//i14

[10] Bernstein D. J., Cache-timing attacks on aes, Technikac report (2005).

[11] Intel, Advanced vector extensions programming reference, http://software.intel.com/file/35247/ (2008)

[12] Xie T., Feng D., Construct md5 collisions using just a single block of message, Cryptology ePrint Archive, Report 2010/643 (2010), http://eprint.iacr.org/2010/643

[13] Manuel S., Classification and generation of disturbance vectors for collision attacks againdt sha-1, Cryptology ePrint Archive, Report 2008/469 (2008);

http://eprint.iacr.org/2008/469 